\title{
Letter re: Comparison of acetabular and femoral morphologies on hip, pelvic, and lumbar radiographs (Yun et al.)
}

B. Snaith ${ }^{a, b}$

Kevin Flintham ${ }^{a}$

a. Mid Yorkshire Hospitals NHS Trust, Aberford Road, Wakefield, WF1 4DG, United Kingdom

b. University of Bradford, Richmond Road, Bradford, BD7 1DP, United Kingdom

Corresponding author.b.snaith@bradford.ac.uk

We read with interest the recent article by Yun et al. [1] comparing acetabular and hip measurements across pelvis, hip and lumbar spine radiographs. The authors assert that lumbar radiographs can be utilised in place of routine pelvis radiographs for these measurements. The example lumbar spine radiograph (figure 2 ) appears to be an abdominal image, with a contrast urogram. Indeed, standard texts $[2,3]$ confirm that the anteroposterior lumbar spine radiograph should not include any coverage of the hips as appropriate collimation should limit the anatomy to T12 superiorly, lower sacrum inferiorly and the sacroiliac joints laterally, which would exclude the hip joints. Thus assessing any hip measurements on an appropriately collimated lumbar spine radiograph should not be possible. This is further compounded by the description of the centring point within their study (iliac crest), which varies from the internationally recognised standard of lower costal margin/L3 [2,3].

The article also states that patients were excluded where the pelvic tilt exceeded the levels recommended by Siebenrock et al. [4]. Was the measure of pelvis tilt, and therefore exclusion, based only on the pelvic radiographs? Imaging of the lumbar spine with the knees flexed, as is usual positioning, will change the pelvic tilt and could lead to inappropriate exclusion if these images were used. Further, this position will likely lead to inaccurate measures due to the retroversion of the acetabulum expected with an increased anterior pelvic tilt/reduced lumbar lordosis. The images used in figure 1 of the article demonstrate a variety of radiographs collimated to show the hip joint but in each case prominence of the ischial spine (PRIS) confirms that the pelvic tilt will have exceeded the limits set within the study [5].

We also found it interesting that of the final diagnoses of the 101 patients within the study there was no mention of degenerative changes, as would be expected in this age group. Was this diagnosis deliberately excluded or classified as 'normal'?

Finally, the authors assert that lumbar spine radiographs can be utilised to perform pathomorphology related measurements of the hip and thus reduce radiation dose. However, in their results the measurements for acetabular roof angle of Tonnis, center-edge angle of Wiberg and femoral head extrusion index differ, to a level of statistical significance, between pelvis and lumbar spine radiographs for all readers (and both anatomical sides) in 11 of the 18 measures. Although this has been described within the highlights of the article, the main conclusion appears to ignore these results.

\section{Declarations of interest}

None.

\section{References}

[1] Yun SJ, Shin YK, Park JS, Jin W, Park YS, Park SY, et al. Comparison of acetabular and femoral morphologies on hip, pelvic, and lumbar radiographs.

doi:https://doi.org/10.1016/j.clinimag.2018.06.003 
[2] Martensen K. McQuillen, Radiographic image analysis, 4th ed., 2015, Saunders; St Louis.

[3] E. Carver and B. Carver, Medical imaging, 2nd ed., 2012, Churchill Livingstone.

[4] K.A. Siebenrock, D.F. Kalbermatten and R. Ganz, Effect of pelvic tilt on acetabular retroversion: a study of pelves from cadavers, Clin Orthop Relat Res 407, 2003, 241-248.

[5] F. Kalberer, S.S. Maden and R. Ganz, Ischial spine projection into the pelvis, Clin Orthop Relat Res 466, 2008, 677-683. 